\title{
Fatores associados à aceitação dos medicamentos genéricos pela população
}

\author{
Factors associated with the acceptance of generic medicines by the population \\ Factores associados a la aceptación de medicamentos genéricos por la población
}

Recebido: 19/07/2021 | Revisado: 27/07/2021 | Aceito: 30/07/2021 | Publicado: 05/08/2021

\author{
André Fabricio Pereira da Cruz \\ ORCID: https://orcid.org/0000-0003-3289-9121 \\ Universidade Federal de Minas Gerais, Brasil \\ E-mail: andrefabriciocruz@yahoo.com.br \\ Aniely Soares Balieiro \\ ORCID: https://orcid.org/0000-0002-0102-3218 \\ Faculdade de Saúde Ibituruna, Brasil \\ E-mail: anielybalieiro@gmail.com \\ Josélia Braz Cruz \\ ORCID: https://orcid.org/0000-0001-5807-4692 \\ Faculdade de Saúde Ibituruna, Brasil \\ E-mail: joseliavgmg@ hotmail.com \\ Artur de Melo Neves \\ ORCID: https://orcid.org/0000-0002-0349-3707 \\ Faculdade de Saúde Ibituruna, Brasil \\ E-mail: arturneves123@outlook.com \\ Pedro Henrique Pereira Costa \\ ORCID: https://orcid.org/0000-0003-4797-8148 \\ Faculdade de Saúde Ibituruna, Brasil \\ E-mail: peu_rpm@hotmail.com
}

\begin{abstract}
Resumo
Objetivo: Verificar a aceitação da população na aquisição de medicamentos genéricos e os fatores que podem estar associados. Métodos: Trata-se de um estudo de caráter descritivo, conduzido com 393 indivíduos, de ambos os sexos, idade de 18 a 80 anos. Os usuários foram investigados quanto a aceitação e utilização dos medicamentos genéricos. Os dados obtidos foram introduzidos em planilha do software Excel versão 2013 e transportados aos softwares IBM® SPSS ${ }^{\circledR}$ Statistics versão 24.0. O estudo foi aprovado pelo Comitê de Ética em Pesquisa. Resultados: Evidenciou-se que a maioria dos entrevistados $(96,5 \%)$ afirmaram já ter utilizado algum medicamento genérico e 89,8 relatam ter atingido resultado eficaz nos tratamentos realizados com esses medicamentos e que após a utilização sentiu que houve o efeito desejado. Conclusão: Os medicamentos genéricos têm aceitação pela maior parte da população. E o grau de conhecimento sobre os medicamentos genéricos pela população aumentou. A população confia no farmacêutico para realizar a troca do medicamento de marca pelo genérico.
\end{abstract}

Palavras-chave: Medicamentos genéricos; Aceitação; População.

\begin{abstract}
Objective: To verify the acceptance of the population in the purchase of generic drugs and the factors that may be associated. Methods: This is a descriptive study, conducted with 393 individuals, of both genders, aged between 18 and 80 years. Users were investigated for acceptance and use of generic drugs. The data obtained were entered into an Excel version 2013 spreadsheet and transferred to the IBM® SPSS $®$ Statistics version 24.0 software. The study was approved by the Research Ethics Committee. Results: It was evident that the majority of respondents (96.5\%) said they had already used a generic drug and 89.8 reported having achieved an effective result in the treatments performed with these drugs and that after use they felt that there was the desired effect. Conclusion: Generic drugs are accepted by most of the population. And the level of knowledge about generic drugs by the population has increased. The population trusts the pharmacist to switch from the branded drug to the generic one.
\end{abstract}

Keywords: Generic drugs; Acceptance; Population.

\section{Resumen}

Objetivo: Verificar la aceptación de la población en la compra de medicamentos genéricos y los factores que pueden estar asociados. Métodos: se trata de un estudio descriptivo, realizado con 393 individuos, de ambos sexos, con edades comprendidas entre 18 y 80 años. Se investigó a los usuarios para determinar la aceptación y el uso de medicamentos genéricos. Los datos obtenidos se ingresaron en una hoja de cálculo de Excel versión 2013 y se transfirieron al software IBM® SPSS® Statistics versión 24.0. El estudio fue aprobado por el Comité de Ética en Investigación. Resultados: Se evidenció que la mayoría de los encuestados $(96,5 \%)$ dijeron que ya habían usado un medicamento genérico y 89,8 reportaron haber logrado un resultado efectivo en los tratamientos realizados con estos medicamentos 
y que luego de su uso sintieron que existía el efecto deseado. Conclusión: Los medicamentos genéricos son aceptados por la mayoría de la población. Y ha aumentado el nivel de conocimiento sobre medicamentos genéricos por parte de la población. La población confía en el farmacéutico para cambiar el medicamento de marca por uno genérico.

Palabras clave: Medicamentos genéricos; Aceptación; Población.

\section{Introdução}

O medicamento é produto farmacêutico, tecnicamente obtido ou elaborado, com finalidade profilática, curativa, paliativa ou para fins de diagnóstico (Agência Nacional de Vigilância Sanitária (ANVISA), 2020).

Os medicamentos são classificados em três classes: referência, similar e genérico. Sendo assim definidos: $O$ medicamento de referência é chamado de produto inovador. Esse medicamento precisa comprovar cientificamente a sua eficácia, qualidade e segurança para poder ser aceito e ser registrado ao órgão federal competente. O medicamento similar é correspondente ao medicamento inovador que foi registrado no órgão federal competente. Deve ser reconhecido pela marca e o seu nome comercial. Ele tem a mesma posologia, indicação terapêutica, dose, princípios ativos, via de administração (Silva et al., 2013).

O que pode ser diferenciado do medicamento de referência são as datas de fabricação, validade, forma do produto, embalagem e tamanho. O medicamento genérico passa pelos mesmos testes científicos que o de referência, ele apresenta a mesma eficácia, qualidade, segurança, mesmas características e efeitos do produto inovador, tem o mesmo princípio ativo, forma farmacêutica, dose e via de administração, possibilitando assim sua intercambialidade com o medicamento de referência. A sua embalagem leva o nome do princípio ativo do medicamento e uma faixa amarela com a letra $\mathrm{G}$ e descrita com o nome "medicamento genérico" (Agência Nacional de Vigilância Sanitária, 2020; Luppe et al., 2020).

Mesmo com a criação de drogas de alta tecnologia, mais potente e de ação cada vez mais específica, a maioria da população mundial estava sem acesso a medicamentos essenciais. Diante disso, essa situação foi ficando cada vez mais preocupante, buscando a necessidade de resolver esse problema, houve assembleias e fóruns de debates em vários países. Onde foi criado a política nacional de medicamentos, destacando a assistência farmacêutica e estratégias para a promoção do acesso a medicamentos. Foi recomendado pela Política Nacional de Medicamentos a adoção de uma política de medicamentos genéricos como uma forma de oferecer medicamentos mais baratos e com qualidade (Silva \& Rocha, 2016; Barata-Silva et al., 2017).

A partir da Lei $n^{\circ} 9787 / 99$, surgiram os medicamentos genéricos. Esses possuem os mesmos princípios ativos e semelhanças do medicamento de referência; e, inclusive, passam por testes de bioequivalência e de qualidade. Os medicamentos genéricos podem ser identificados pela sua tarja de cor amarela, na qual vem escrito "Medicamento genérico". Na embalagem vem o nome do princípio ativo de cada medicamento, pois o genérico não tem um nome ou marca (Silva \& Rocha, 2016).

A garantia da qualidade do medicamento genérico se dá a partir da avaliação pela ANVISA pelos resultados dos testes de bioequivalência, se não houver nenhuma diferença de acordo o de referência em relação a velocidade e extensão de absorção do medicamento, expressa que são intercambiáveis. A diferença na forma de produção, que pode ocorrer por conta de equipamentos diferentes e diferentes produtores de matéria prima, não devem interferir na bioequivalência entre os produtos (Alonso et al., 2015; Barata-Silva et al., 2017).

Os surgimentos dos genéricos veio para promover uma concorrência no setor, trazendo novas opções de medicamentos a população. A possibilidade de substituição do medicamento de referência pelo medicamento genérico, proporcionou a população uma redução nos preços e as indústrias obtiveram uma maior produção de genéricos (Nishijima et al., 2014). 
Os medicamentos genéricos estão sendo cada vez mais aceitos pela população. Devido seu baixo custo, eles passaram a ser comercializados com maior frequência. Pelo fato de gastos em saúde estarem aumentando progressivamente, os medicamentos de referência obtiveram um acesso bastante restrito pelo seu custo mais elevado, tornando assim o acesso aos genéricos mais contínuo (Fernandes et al., 2011).

O medicamento genérico tem um baixo custo por já existir um medicamento igual a ele no mercado, não precisando investir em estudo para comprovar sua eficácia. E como o genérico não tem nome comercial, não tem investimentos em propaganda, diminuindo gastos com o medicamento (Agência Nacional de Vigilância Sanitária, 2020).

Esses medicamentos são aproximadamente $35 \%$ mais barato que os medicamentos de referência, garantindo a mesma eficácia aos seus usuários e um menor valor a ser gasto. Além dessa vantagem, os medicamentos genéricos vendidos no Brasil, corresponde cerca de 34\% e 85\% dos medicamentos dispensados no Programa Farmácia Popular do Brasil, são medicamentos genéricos. Dessa forma, observa- se que a aceitação está crescendo gradativamente e acesso a população está maior (Pinheiro da Silva et al., 2020).

Com o avanço da tecnologia, o conhecimento sobre o genérico está cada vez maior, assim, fazendo os usuários ter uma confiabilidade e passando a comprar mais esse medicamento, sem contar o papel que o farmacêutico vem fazendo, orientando os usuários quanto a qualidade e eficácia dos mesmos e promovendo o uso racional de medicamentos. Essa estratégia ajuda na aceitação e utilização, pois ainda existem muitas pessoas que não tem esse conhecimento sobre a mesma eficácia de ambos (Guttier et al., 2016).

Além disso o farmacêutico é um profissional habilitado para poder fazer a troca de medicamentos equivalentes para o genérico, ou seja, a intercambialidade. Essa troca pode ocorrer no ato da compra do medicamento, caso o paciente desejar substituir e/ou o farmacêutico lhe apresentar essa ideia de substituição, contanto que o medicamento seja substituído por um medicamento correspondente ao de referência. Só não poderá ser substituído caso o médico redigir na receita que não poderá substituir esse medicamento (Nardi \& Ferraz, 2016; Leal et al., 2017).

Embora os medicamentos genéricos ainda não sejam muitos prescritos pelos médicos, essa perspectiva está mudando, intervenções estão sendo feitas e com o passar do tempo os médicos estão tendo mais confiabilidade nesses medicamentos e estão mudando a sua conduta nas prescrições médicas, e assim, promovendo o uso racional de medicamentos (Guttier et al., 2016).

Diante disso, o intuito deste estudo foi avaliar os fatores associados a aceitação dos medicamentos genéricos pela população.

\section{Metodologia}

Este estudo possui caráter descritivo, de corte transversal e análise quantitativa de campo, que objetivou avaliar aceitação da população na aquisição de medicamentos genéricos e os fatores que podem estar associados. A amostra do estudo, foi constituída, a partir de cálculo amostral, por 393 participantes de municípios de todo o Brasil que obtiveram acesso ao Google Forms, de ambos os sexos, com faixa etária de 18 aos 80 anos. A coleta de dados foi realizada através do preenchimento de um questionário, de forma individualizada, com perguntas objetivas de múltipla escolha, inerentes ao objetivo da pesquisa, sem a necessidade de identificação do voluntário. O questionário foi aplicado, somente, para aqueles que aceitaram em participar da pesquisa e assinaram o Termo de Consentimento Livre e Esclarecido (TCLE). E foram excluídos da pesquisa o público que não conseguiram responder o questionário completo, pessoas que nunca utilizaram os medicamentos genéricos, indivíduos que recusarem assinar o TCLE e menores de 18 anos. O questionário avaliou à aceitação, confiança e utilização dos medicamentos genéricos pela população. 
Os dados coletados foram reunidos, armazenados em planilha no Software Excel 2013 e avaliados estatisticamente no software IBM® SPSS® Statistics versão 24.0 bases de análise estatística for Windows. Foi utilizado o teste Qui-quadrado para avaliar as diferenças de proporções dos dados categóricos sendo considerado significativo $\mathrm{p} \leq 0,05$.

O estudo foi elaborado seguindo as normas e diretrizes definidas pelas Resoluções do Conselho de Saúde que regulamentam a realização de pesquisa envolvendo seres humanos no 466/2012 e $n^{\circ}$ 510/2016 e submetido na plataforma Brasil, com aprovação pelo Comitê de Ética em Pesquisa envolvendo Seres Humanos das Faculdades Unidas do Norte de Minas - FUNORTE, sob o número do parecer 4.494.208. Além disso, foram respeitados os preceitos éticos e os pesquisadores assinaram o termo de proteção de risco e confidencialidade comprometendo-se a guardar sigilo das informações contidas nos prontuários e prescrições médicas e utilizá-los apenas para fins científicos.

\section{Resultados}

Foram entrevistados um total de 393 indivíduos de ambos os sexos. Dos entrevistados, 33,3\% eram do sexo masculino e $66,7 \%$ do sexo feminino. E, em relação a idade, 63,8\% dos entrevistados tinham idade entre 18 e 30 anos; $26,5 \%$ compreenderam indivíduos com idade entre 31 e 45 anos; 9,2\% com idade entre 46 e 60 anos e 0,5\% dos entrevistados tinham idade superior a 60 anos. Em se tratando da escolaridade, houve uma predominância de indivíduos com ensino médio incompleto, correspondendo a 44,9\%, 24,2\% tinham superior completo e apenas 3,3\% dos entrevistados possuíam ensino médio completo. De acordo à renda dos entrevistados, constatou-se que a maior parte deles $(31,8 \%)$, possuem renda familiar de até 01 salário mínimo, 28,8\% nenhuma renda, 28\% está entre 01 e 03 salários, 11,5\% acima de 3 salários. Em todas as avaliações sociodemográficas, obteve-se diferença significativa entre as variáveis com $\mathrm{p} \leq 0,05$ (Tabela 1).

Tabela 1 - Distribuição das características sociodemográficas da população.

\begin{tabular}{|c|c|c|c|c|}
\hline PERFIL & SOCIODEMOGRÁFICO & $\begin{array}{c}\text { FREQUENCIA } \\
\text { ABSOLUTA }\end{array}$ & $\begin{array}{c}\text { FREQUENCIA } \\
\text { RELATIVA }\end{array}$ & Sig \\
\hline \multirow{2}{*}{ Gênero } & Feminino & 262 & 66,7 & \multirow{2}{*}{$0,000 *$} \\
\hline & Masculino & 131 & 33,3 & \\
\hline \multirow{4}{*}{ Idade } & 18 a 30 anos & 250 & 63,8 & \multirow{4}{*}{$0,000^{*}$} \\
\hline & 31 a 45 anos & 104 & 26,5 & \\
\hline & 46 a 60 anos & 36 & 9,2 & \\
\hline & Mais de 60 anos & 2 & 0,5 & \\
\hline \multirow{6}{*}{ Escolaridade } & Fundamental incompleto & 7 & 1,8 & \multirow{6}{*}{$0,000 *$} \\
\hline & Fundamental completo & 9 & 2,3 & \\
\hline & Ensino médio incompleto & 176 & 44,9 & \\
\hline & Ensino médio completo & 13 & 3,3 & \\
\hline & Superior incompleto & 92 & 23,5 & \\
\hline & Superior completo & 95 & 24,2 & \\
\hline \multirow{4}{*}{ Renda Mensal } & Nenhuma renda & 113 & 28,8 & \multirow{4}{*}{0,000} \\
\hline & Até 01 salário mínimo & 125 & 31,8 & \\
\hline & Entre 01 e 03 salários & 110 & 28 & \\
\hline & Acima de 03 salários & 45 & 11,5 & \\
\hline
\end{tabular}

Fonte: Autores (2021).

Dos 393 entrevistados, 96,5\% afirmam já ter utilizado algum medicamento genérico e 89,8\% relatam ter atingido resultado eficaz nos tratamentos realizados com esses medicamentos e que após a utilização sentiu que houve o efeito desejado (Gráfico 1 e 2). 
Gráfico 1 - Utilização de medicamentos genéricos pela população.

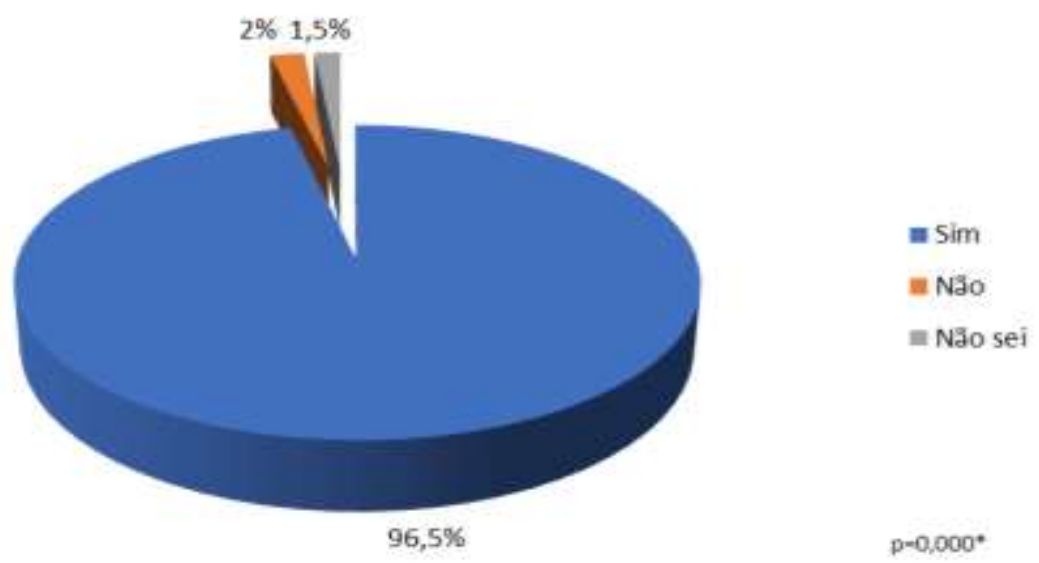

Fonte: Autores (2021).

Gráfico 2 - Classificação da população se houve o efeito desejado após tomar o medicamento genérico.

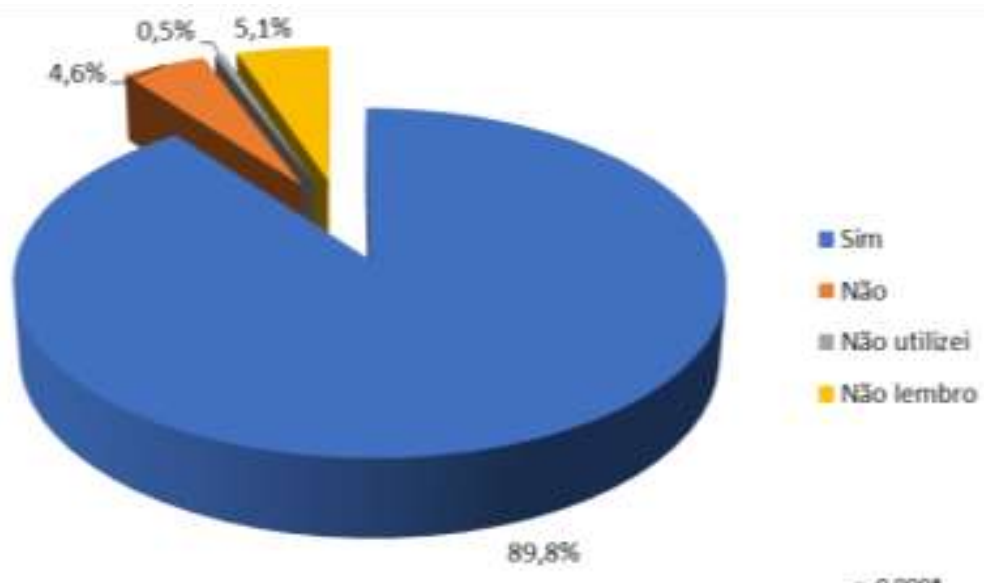

Fonte: Autores (2021).

A minoria dos participantes $(35,1 \%)$, relataram que ao ir ao médico pede para poder receitar medicamento genérico, já $64,9 \%$ relataram que não fazem o pedido dessa prescrição (Tabela 2).

Sobre à confiança que o usuário possui no farmacêutico para realizar a troca do medicamento de marca pelo genérico correspondente, a maioria dos participantes $(86,8 \%)$ aceitam que o farmacêutico faça a troca, enquanto $12,5 \%$ não aceitam que o farmacêutico faça a troca.

Quando questionados se acredita que o medicamento genérico tenha a mesma eficácia que o de marca, 85,2\% responderam que sim e 14,8\% disseram que não. 
Tabela 2 - Informações sobre os medicamentos genéricos.

\begin{tabular}{lcccc}
\hline & & $\mathbf{n}$ & $\%$ & Sig \\
\hline Quando vai ao médico pede para ele & Sim & 138 & 35,1 & $0,000^{*}$ \\
receitar medicamento genérico & Não & 255 & 64,9 & \\
\hline & Sim & 341 & 86,8 & $0,000^{*}$ \\
Confiaria um farmacêutico fazer a troca & Não & 49 & 12,5 & \\
de um medicamento de marca por um & Não utilizo & & & \\
genérico & medicamento & 3 & 0,7 & $0,000^{*}$ \\
\hline Acredita que o medicamento genérico & genérico & Sim & 334 & 85,2 \\
tenha a mesma eficácia que o de marca & Não & 58 & 14,8 & \\
\hline
\end{tabular}

Fonte: Autores (2021).

Foi analisado à associação entre a escolaridade e a confiança na eficácia do medicamento genérico, observou-se que houve a associação $(\mathrm{p}=0,021)$. Pode-se notar que $100 \%$ dos entrevistados que tem o ensino fundamental incompleto e ensino médio completo, diz confiar na eficácia, quanto ao ensino médio incompleto, 94,9\%, sendo a maioria, também diz confiar na eficácia do genérico. Entretanto $11,1 \%$ do ensino fundamental completo não confia na eficácia dos medicamentos genéricos (Tabela 3).

Tabela 3 - Comparação entre a escolaridade e confiança na eficácia do medicamento genérico.

\begin{tabular}{|c|c|c|c|c|c|c|c|}
\hline \multirow{3}{*}{ Escolaridade } & \multicolumn{7}{|c|}{ Confia na eficácia do medicamento genérico } \\
\hline & \multicolumn{2}{|c|}{ Sim } & \multicolumn{2}{|c|}{ Não } & \multicolumn{2}{|c|}{$\begin{array}{c}\text { Não sei } \\
\text { informar }\end{array}$} & \multirow[t]{2}{*}{ Sig } \\
\hline & $\mathbf{n}$ & $\%$ & $\bar{n}$ & $\%$ & $\bar{n}$ & $\%$ & \\
\hline Fundamental incompleto & 7 & 100,0 & 0 & 0,0 & 0 & 0,0 & \multirow{6}{*}{$0,021 *$} \\
\hline Fundamental completo & 6 & 66,7 & 1 & 11,1 & 2 & 22,2 & \\
\hline Ensino médio incompleto & 167 & 94,9 & 6 & 3,4 & 3 & 1,7 & \\
\hline Ensino médio completo & 13 & 100,0 & 0 & 0,0 & 0 & 0,0 & \\
\hline Superior incompleto & 78 & 84,8 & 6 & 6,5 & 8 & 8,7 & \\
\hline Superior completo & 87 & 91,6 & 1 & 1,1 & 7 & 7,4 & \\
\hline
\end{tabular}

Fonte: Autores (2021).

Foi analisado à associação entre a renda mensal e o principal motivo para escolher o medicamento genérico, e houve a associação entre eles $(\mathrm{p}=0,002)$. Pode- se notar que a predominância foi de acordo ao preço e a renda mensal que mais escolhe pelo preço são os que tem renda acima de 3 salários mínimos $(77,8 \%)$ e de renda entre 01 e 03 salários $(75,5 \%)$ Os entrevistados que diz não ter nenhuma renda (19,5\%), diz que escolhe o medicamento genérico pela qualidade, 20,4\% já relata que escolhe o medicamento pela facilidade de encontrar e 57,5\% já prefere pelo preço (Tabela 4). 
Tabela 4 - Associação da renda mensal entre qual o principal motivo para escolher o medicamento genérico.

\begin{tabular}{|c|c|c|c|c|c|c|c|c|c|}
\hline \multirow{3}{*}{ Renda mensal } & \multicolumn{9}{|c|}{ Qual o principal motivo para escolher o medicamento genérico } \\
\hline & \multicolumn{2}{|c|}{$\begin{array}{c}\text { Não uso } \\
\text { medicamento } \\
\text { genérico }\end{array}$} & \multicolumn{2}{|c|}{ O preço } & \multicolumn{2}{|c|}{ A qualidade } & \multicolumn{2}{|c|}{$\begin{array}{l}\text { A facilidade } \\
\text { de encontrar }\end{array}$} & \multirow[t]{2}{*}{ Sig } \\
\hline & $\mathbf{n}$ & $\%$ & $\mathbf{n}$ & $\%$ & $\mathbf{n}$ & $\%$ & $\mathbf{n}$ & $\%$ & \\
\hline Nenhuma renda & 3 & 2,7 & 65 & 57,5 & 22 & 19,5 & 23 & 20,4 & \multirow{4}{*}{$0,002 *$} \\
\hline Até 01 salário mínimo & 4 & 3,2 & 93 & 74,4 & 7 & 5,6 & 21 & 16,8 & \\
\hline Entre 01 e 03 salários & 0 & 0,0 & 83 & 75,5 & 7 & 6,4 & 20 & 18,1 & \\
\hline Acima de 03 salários & 0 & 0,0 & 35 & 77,8 & 1 & 2,2 & 9 & 20,0 & \\
\hline
\end{tabular}

Fonte: Autores (2021).

\section{Discussão}

Neste trabalho, verificou-se que as mulheres consomem mais medicamentos genéricos que os homens. De acordo com Bertoldi, Arrais, Tavares, Ramos, Luiza, Mengue, Dal-Pizzol, Farias e Oliveira (2016), a prevalência do uso de medicamentos é mais alta entre as mulheres, e isso pode estar relacionado com o fato das mulheres, desde à adolescência, já utilizarem mais medicamentos que os adolescentes. Por iniciarem a utilizar o uso de medicamentos precocemente, conhecem mais sobre os medicamentos genéricos, e por esse motivo consomem mais. Esse fato não está relacionado com o sexo e sim com o maior tempo de consumo de medicamentos entre as mulheres.

Com os dados obtidos, pode-se observar que a maioria dos participantes já utilizaram o medicamento genérico pelo menos uma vez na vida, deixando evidente que há uma grande aceitação desses medicamentos. De acordo com os estudos de Da Silva, Valiatti, Calazans, Barcelos e De Oliveira Salvi (2017), verificou que a maioria dos participantes compraram os medicamentos genéricos com a receita médica, porém, não significa que nessas receitas estavam receitados, genéricos, pois no momento da compra o farmacêutico pode ter feito a intercambialidade. Destaca-se que aspectos como o fácil acesso e o menor preço, comparado com os medicamentos referência, podem influenciar neste número significativo de pessoas que já fizeram uso de medicamento genérico.

Os dados deste trabalho, também corroboram com achados de Alcântara (2018), que em sua pesquisa também verificou que a maioria dos entrevistados fizeram o uso de medicamentos genéricos e que, além disso, obtiveram o resultado farmacológico esperado. Essa equivalência na resposta farmacoterapêutica é esperada uma vez que o medicamento genérico apresenta a mesma composição, as mesmas propriedades farmacocinéticas e farmacodinâmicas a fim de garantir as mesmas características do medicamento de referência.

A maioria dos entrevistados dizem não pedir para o médico receitar o medicamento genérico. Em um estudo feito por Duque, Rocha e Balteiro (2014), a maioria também não faz essa solicitação, visto que os pacientes afirmam que essa decisão tem que ser tomada apenas pelo médico. No entanto, é de suma importância que o prescritor incentive o uso do medicamento genérico, já que o mesmo, possui a mesma eficácia do medicamento referência, com um menor custo para o usuário que pode fazer uso dessa economia, com outras necessidades pessoais. Ademais, reduzir o custo de determinado tratamento pode contribuir em uma maior adesão do paciente ao tratamento, principalmente, e farmacoterapias de uso crônico.

Estudos similares apontam que o farmacêutico realiza a substituição do medicamento genérico, que foi prescrito pelo médico. A intercambiação só não pode ser realizada se o próprio médico restringir a troca. O farmacêutico, portanto, pode dispensar o medicamento de referência quanto o medicamento genérico e similares do original (Lima et al., 2020). 
Alguns participantes disseram que não sentiu que surtiu o efeito desejado. Isso pode estar relacionado com o comportamento do paciente que, talvez não tenha utilizado o medicamento corretamente e/ou não tenha tomado esse medicamento anteriormente, criando assim uma perspectiva inexistente em relação ao fármaco. Ademais, essa ineficácia pode não ser uma resposta especificamente e exclusiva do medicamento genérico. Lira, Oliveira, Andrade, Vancini-Campanharo e Vancini (2014), retrataram que essa perda de eficácia e variabilidade desses efeitos podem existir e estarem relacionados com fatores que estão ligados ao paciente como: idade, outras doenças e gravidez, que podem gerar efeitos e respostas terapêuticas diferentes do que se espera.

A maioria dos participantes relataram que confiam na substituição do medicamento de marca pelo genérico através da ação do farmacêutico. Essa confiança, está crescendo ao longo do tempo, e outros estudos evidenciaram que $86 \%$ dos entrevistados confiam no farmacêutico para a realização da substituição (Teixeira et al., 2017). O aumento desta confiança pode estar inerente a maior difusão de informações a respeito dos medicamentos genéricos.

Sabe-se que o farmacêutico junto com o médico devem ser os maiores incentivadores do uso de medicamentos genéricos, buscando sempre passar informações corretas em relação a estes medicamentos para os pacientes, pois na maioria das vezes são pessoas com pouco ou nenhum entendimento do assunto, tomando, consequentemente, suas decisões sobre qual medicamento comprar, diante da orientação desses profissionais (Silva et al., 2013). Esse comportamento instrutivo, é de suma importância, já que dependendo do medicamento de marca prescrito, o valor pode ser significativamente maior. Assim, possibilitar a intercambialidade por um fármaco com a mesma eficácia e com custo menor, pode garantir a adesão do paciente ao tratamento e a transferência desses recursos economizados para outras finalidades pessoais.

Quando questionados se acredita que o medicamento genérico tenha a mesma eficácia que o de marca, a maioria dos entrevistados disseram que sim. Resultados semelhantes foram encontrados em um estudo realizado por Lira et al. (2014), que também indica que a maioria acredita na eficácia dos medicamentos genéricos. Outro estudo também corrobora que a grande maioria dos usuários também acreditam que o medicamento genérico produz o mesmo efeito que o de referência no organismo. Conforme o estudo realizado pela Pro-genéricos, retrata que 65\% das prescrições que são receitadas, equivale a medicamentos genéricos. Isso comprova que a população está tendo mais credibilidade, com o aumento da utilização e a confiança dos medicamentos genéricos (World Health Organization (WHO), 2014; Alcântara, 2018).

Dos participantes desta pesquisa, 14,8\% disseram que o medicamento genérico não tem a mesma eficácia. Esse aspecto pode ser devido à falta de informações sobre os medicamentos genéricos. Sabe-se que os mesmos passam pelos mesmos testes científicos que os de referência, contendo a mesma eficácia, qualidade e segurança (Machado et al., 2020).

Pode ser observado que os entrevistados confiam na eficácia do medicamento, independente da sua escolaridade. Os meios de comunicação têm contribuído, seja pela televisão, rádio, internet, pelos farmacêuticos e a própria população que passa de um para outro, fazendo com que os medicamentos genéricos fiquem cada vez mais conhecidos, e assim uma pessoa com qualquer grau de escolaridade tem o conhecimento sobre eles.

De acordo com Fernandes, Coutinho e Valle (2011), pode-se observar que grande parte dos usuários de medicamentos genéricos, escolhem esse produto devido seu baixo valor comercial. Outro dado a ser observado, que independente da renda, os participantes se importam com o valor do medicamento. No momento da compra sempre buscam pelo medicamento com baixo preço, mostrando sim que o preço é um fator considerável na hora da compra.

O preço do medicamento pode influenciar a decisão do paciente, por ser o mesmo medicamento com um preço melhor, chama mais atenção da população e preferem comprar o medicamento genérico (Han et al., 2001; Xavier et al., 2019). E a qualidade do medicamento genérico é feita por testes científicos que comprovam a mesma eficácia e qualidade do medicamento de referência. 


\section{Conclusão}

Os dados analisados permitiram concluir que os medicamentos genéricos têm aceitação pela maior parte dos participantes. Constatou-se que o grau de conhecimento sobre os medicamentos genéricos pela população aumentou. Além disso, a população mostrou ter confiança nos resultados obtidos ao fazer uso destes, houve predominância na busca desses medicamentos, por conta do preço se comparando com o medicamento de referência. A maioria dos participantes confiam no farmacêutico para realizar a troca do medicamento de marca pelo genérico, uma vez que a qualidade desse medicamento se dar pela realização dos testes, onde comprovam a mesma eficácia e qualidade do medicamento de referência. Os farmacêuticos estão sempre orientando a população, levando sempre a informação correta quanto ao uso do genérico.

\section{Referências}

Agência Nacional de Vigilância Sanitária (ANVISA). Ministério da Saúde. Medicamentos Genéricos. 2020. http://www.anvisa.org.br.

Alcântara, R. F. (2018). A percepção da população de consumidores de medicamentos genéricos em farmácias comerciais na região metropolitana do cariri. Journal of Biology \& Pharmacy and Agricultural Management, 13(4).

Alonso, M. A. S., Barbosa, A. F., Silva, N. G. C., Cruz, K. de M. B., Silva, J. R. G., de Mattos Machado, S. H., \& Silva, D. A. (2015). Adesão à prescrição de medicamentos genéricos por parte dos profissionais da área médica. Acta Biomedica Brasiliensia, 6(1), 45-55.

Barata-Silva, C., Hauser-Davis, R. A., Silva, A. L. O. da, \& Moreira, J. C. (2017). Desafios ao controle da qualidade de medicamentos no Brasil. Cadernos Saúde Coletiva, 25, 362-370.

Bertoldi, A. D., Arrais, P. S. D., Tavares, N. U. L., Ramos, L. R., Luiza, V. L., Mengue, S. S., Dal-Pizzol, T. da S., Farias, M. R., \& Oliveira, M. A. (2016). Utilização de medicamentos genéricos na população brasileira: Uma avaliação da PNAUM 2014. Revista de Saúde Pública, 50.

Da Silva, C. M., Valiatti, T. B., Calazans, R. da S. P., Barcelos, I. B., \& de Oliveira Salvi, J. (2017). Medicamentos genéricos: Uma abordagem no município de Vale do Paraíso, Rondônia. Acta Biomédica Brasiliensia, 8(2), 83-90.

De Amorim Rodrigues, L., de Oliveira Freitas, T., Coelho, V. A. T., de Souza, C. G., \& de Araújo, L. B. S. (2020). Medicamentos genéricos nos últimos 20 anos e a percepção dos consumidores. Revista Multidisciplinar do Nordeste Mineiro, 1, 01.

Duque, M., Rocha, C., \& Balteiro, J. (2014). Adesão dos utentes aos medicamentos genéricos. Revista Portuguesa de Saúde Pública, $32(2), 181-187$.

Fernandes, J. A., Coutinho, J. V., \& Valle, M. do. (2011). Aceitação do medicamento genérico em diferentes níveis de escolaridade e renda familiar do Distrito Federal. Cenario Pharmacêutico, 4(4).

Guttier, M. C., Silveira, M. P. T., Luiza, V. L., \& Bertoldi, A. D. (2016). Percepção, conhecimento e uso de medicamentos genéricos no Sul do Brasil: O que mudou entre 2002 e 2012? Cadernos de Saúde Pública, 32.

Han, S., Gupta, S., \& Lehmann, D. R. (2001). Consumer price sensitivity and price thresholds. Journal of retailing, 77(4), 435-456.

Leal, L. B., de Araujo, T. P., Chagas, S. C. C., Andrade, A. R. B., Bedor, D. C. G., \& Santana, D. P. (2017). Registro de medicamentos genéricos tópicos dermatológicos: Cenário brasileiro e estudos para demonstração de bioequivalência. Vigilância Sanitária em Debate: Sociedade, Ciência \& Tecnologia (Health Surveillance under Debate: Society, Science \& Technology)-Visa em Debate, 5(2), 3-12.

Lima, R. Q., de Almeida, M. C. P., Júnior, E. D. N. F., \& Neto, L. de S. L. (2020). Intercambialidade entre medicamentos de referência e similar. Brazilian Journal of Development, 6(12), 101122-101132.

Lira, C. A. B. de, Oliveira, J. N. S., Andrade, M. dos S., Vancini-Campanharo, C. R., \& Vancini, R. L. (2014). Conhecimento, percepções e utilização de medicamentos genéricos: Um estudo transversal. Einstein (Sao Paulo), 12, 267-273.

Luppe, M. R., Rossi, C. M., Torres, R. R., \& de Souza Aguiar, H. (2020). Análise de atributos na preferência entre consumo de medicamentos genéricos e similares ou medicamentos de referência. Revista Gestão Organizacional, 13(2), 48-66.

Machado, F. E., Braun, E. L., \& Mastella, A. K. (2020). Avaliação da aceitação de medicamentos genéricos e seus desafios no mercado farmacêutico. Anais do Seminário Interinstitucional de Ensino, Pesquisa e Extensão.

Nardi, E. P., \& Ferraz, M. B. (2016). Percepção de valor de medicamentos genéricos em São Paulo, Brasil. Cadernos de Saúde Pública, 32(2).

Nishijima, M., Biasoto Jr, G., \& Lagroteria, E. (2014). A competição no mercado farmacêutico brasileiro após uma década de medicamentos genéricos: Uma análise de rivalidade em um mercado regulado. Economia e sociedade, 23, 155-186. 
Research, Society and Development, v. 10, n. 10, e68101018438, 2021

(CC BY 4.0) | ISSN 2525-3409 | DOI: http://dx.doi.org/10.33448/rsd-v10i10.18438

Pinheiro da Silva, A. E., Bonfim, A. J., \& Barroso Oliveira, F. C. (2020). Utilização de medicamentos genéricos em um estabelecimento farmacêutico do município de Teresina (PI). e-Revista Facitec, 11(1).

Silva, N. C. S., \& Rocha, L. C. (2016). Medicamentos genericos: Legislacao, politica e mercado. Unica Cadernos Academicos, 3(1).

Silva, J. L. et al. (2013). Avaliação da dispensação de medicamentos genéricos em farmácias privativas de Maringá-PR. Brasilian Journal of Surgery and Clinical Research; 4(3): 5-8.

Teixeira, K. B., Ribeiro, M. S., Lafetá, B. N., Tourinho, F. M., \& de Paula, L. R. E. S. ([s.d.]). Avaliação do perfil de utilização dos medicamentos genéricos. Revista Eletrônica Acervo Saúde/ElectronicJournalCollection Health

Xavier, J. L. S., Mendes, M. M. V., dos Santos, T. A. X., \& Borges, B. K. A. (2019). Conhecimento e utilização de medicamentos genéricos, similares e de referência por pacientes em unidades básicas de saúde de montes claros-mg. Revista Uningá, 56(1), 197-204.

World Health Organization (WHO) (2021). Pró-Genéricos (2014) Genéricos são 65\% dos medicamentos mais prescritos no país, diz entidade. http://www.progenericos.org.br/index.php/noticias/361-mercado-aberto-genericos-sao-65-dos-medicamentos-mais-prescritos-no-pais-diz-entidade-folha-despaulo. 